\title{
TOWARDS THREE-DIMENSIONAL MODELLING OF SUBGRAIN COALESCENCE
}

\author{
T. O. SAETRE \\ Agder College, Faculty of Engineering, N-4890 Grimstad, Norway
}

(Received 18 January 1996)

$\mathrm{Hu}(1962,1963)$ suggested that the nucleation process during recrystallisation can arise from subgrain coalescence. In the present paper, a new modelling approach has been presented for subgrain rotations. It is suggested that the required mass transfer due to compatibility criteria, can be modelled as geometric friction forces. By employing this new concept, it has been shown that three-dimensional models can be derived, based on classical mechanics. The derived model has shown that the small and regular subgrains are the most likely candidates for rotation. Subgrain coalescence is strongly temperature dependent since diffusion of vacancies are required for the rotation.

KEY WORDS: Coalescence, dislocations, modelling, recovery, subgrains

\section{INTRODUCTION}

Metals which have undergone cold deformation, can be restored to the original mechanical properties by heat treatment. The restoration processes are complex. Although they are of major importance to the manufacturing industries, the industry relies, to a large extent, on empirical data. In textbooks, the restoration is generally described as two different processes; recovery and recrystallisation. In commercial processes it is often seen that recovery and recrystallisation can take place simultaneously in different areas of a specimen and also dynamically during hot deformation. These processes determine the final crystallographic texture and mechanical properties of products and it is thus of importance to be able to make quantitative predictions in order to optimise and control the industrial output.

The term recovery embraces all changes which do not involve the sweeping of the deformed structure by migrating high-angle grain boundaries. Recovery leads to a reduction in the dislocation density, and the remaining dislocations are often found to be in low energy positions as sub-boundaries. The subgrain structure can reduce the energy further by sub-boundary migration, which results in a coarsening of the mean subgrain size by a reduction of the total sub-boundary area. Alternatively, a sub-boundary can obtain a reduction in the specific energy by reducing the angle of misorientation between the neighbouring crystallographic lattices by rotation. This process can ultimately lead to coalescence and consequently, a coarsening of the mean subgrain size. Finally, recrystallisation nuclei are formed and primary recrystallisation results in new, deformation free grains.

It was proposed by $\mathrm{Hu}(1962,1963)$ that coarsening of the subgrain size could take place by coalescence of neighbouring subgrains, due to the disappearance of the dislocations constituting the sub-boundaries between them. Such a process must involve 
both dislocation glide and climb. An enlarged subgrain thus formed, much larger than its neighbours, could then grow at their expense, i.e. act as a recrystallisation nucleus. $\mathrm{Hu}$ (1962) studied the annealing behaviour of Si-Fe single crystals. The experiments, highly advanced at that time, was carried out in-situ in a transmission electron microscope with a heating stage with maximum variation of $\pm 25^{\circ} \mathrm{C}$ for the temperature range $250-800^{\circ} \mathrm{C}$. During such experiments, Hu observed an increase in the average subgrain size, but no migration of the subgrain boundaries. Sub-boundaries were observed to fade away, thus leading to the conclusion that the neighbouring subgrains were rotating towards a common crystallographic orientation. Hu (1962) noted that C. S. Smith had pointed out the possibility of subgrain reorientation in 1948 and that some observations indicating rotating grains, had been reported, cf. references 8 and 9 in $\mathrm{Hu}$ 's paper. Hu considered the possibility of subgrain coalescence as a mechanism for nucleating recrystallisation grains. Based on his model, the incubation period for recrystallisation will correspond to the period for subgrain coalescence, during which two results have to be accomplished: the size of the coalesced subgrains has to become sufficiently large for growth to continue into the matrix, and a high-angle boundary having a high rate of migration due to the higher mobilities of high angle boundaries, has to be developed. By rolling Si-Fe single crystals in the (001)[110] and (001)[100] directions, $\mathrm{Hu}$ (1963) reported recrystallisation behaviour depending strongly on the rolling directions. The (001)[110] crystal did not recrystallise upon annealing, but the (001)[100] crystal was observed to recrystallise easily at moderate temperatures due to the presence of well-defined deformation bands. The microbands (transition bands) were examined by several methods, and orientation differences across microband regions were determined by selected area electron diffraction patterns. Hu concluded that the boundaries of all the microband segments were low-angle boundaries. The orientations of adjacent microbands were related by rotations around both [001] and [110] axes. Consequently, the sub-boundaries could not be simple tilt or twist boundaries, but were highly immobile low angle boundaries consisting of families of dislocations with nonparallel Burgers vectors. Recrystallisation was found to originate within the polygonized microbands. $\mathrm{Hu}$ found indications supporting the hypothesis that coalescence of subgrains leads to the formation of recrystallised grains. Since the mobility of the examined sub-boundaries was generally low, Hu believed, based on the observations, that boundary migration were only a secondary effect and that the growth of subgrains largely occurred by the elimination of boundaries, i.e. by subgrain coalescence. Hu suggested that high-angle boundaries associated with a recrystallised grain could be created by the coalescence process of subgrains in a microband.

Another early work on recovery, where similar observations were made in $\mathrm{Al}$ specimens, was published by Fujita (1961). The process of subgrain rotation was analysed by $\mathrm{Li}$ (1962) from both a thermodynamic and kinetic viewpoint. Li showed, from energetic considerations, that a subgrain can rotate naturally in directions in which low angle boundaries decrease their angles of misorientation to allow high-angle boundaries to increase their angles of misorientation. Twist and asymmetric boundaries can similarly reduce their angles of misorientation by allowing tilt and symmetric boundaries to increase their angles of misorientation. Boundaries of large areas can also reduce the angle of misorientation by allowing smaller area boundaries to increase the angle of misorientation. $\mathrm{Li}$ also showed that either the co-operative diffusion of vacancies or the co-operative movement of dislocations in the boundary can be the rate controlling process, depending on the angle of misorientation of the boundary. 
Since the early works of $\mathrm{Hu}$ and others, a number of papers have been published related to recovery and subgrain coalescence. $\mathrm{Hu}$ (1981) reviewed the state of knowledge of coalescence 20 years later in a brief review on recovery, recrystallisation and grain growth. In particular, $\mathrm{Hu}$ focused in this paper on the scepticism which can be found in part of the scientific community towards subgrain coalescence as an important factor in the nucleation process of recrystallisation. One of the main concerns was the possible uncertainty of the experimental evidence by transmission electron microscopy concerning the disappearance of sub-boundaries in thin foils. Hu concluded that more detailed experimental studies are highly desirable. Moreover, it seemed to be necessary to reexamine the theoretical models since there were a discrepancy between the subgrain growth rate experimentally determined and theoretical predictions. An updated list of references can be found in Saetre and Ryum (1995).

In later years $\mathrm{Hu}$ (1993) was convinced that studying subgrain coalescence by computer simulations is a right approach. $\mathrm{Hu}$ also noted that there has been very little experimental data on subgrain growth in the literature. In his opinion, it should be possible to obtain some good results from experiments by using single crystals. One should preferably use single crystals with stable orientations so that the substructure of the whole sample would be more uniform, and no recrystallisation occurring to interfere the growth of the subgrains. Such stable orientations in rolling are $\{110\}<112>$ for the fcc metals, and $\{100\}<011>$ for the bcc metals. To extend the range of subgrain growth, metals of high melting temperatures and no phase transition could be employed.

\section{MODELLING SUBGRAIN COALESCENCE}

The rotation of an ensemble of three-dimensional subgrains in the bulk of a material during annealing or during hot deformation, requires complex modelling and large computer resources. The general model must allow arbitrary initial crystallographic orientation, morphology and volume of each individual subgrain, surrounded by complex sub-boundaries consisting of families of dislocations with non-parallel Burgers vectors, thus facilitating arbitrary misorientations of neighbouring subgrain lattices. The evolution of each subgrain with details of the sub-boundaries should preferably be available throughout the simulation. In order to make accurate predictions, the evolution of the sub-boundaries should be determined by following groups of dislocations, or individual dislocations when necessary. Migration of the sub-boundaries should be allowed in order to determine the dominating processes during subgrain coarsening: coalescence due to rotations or sub-boundary migration. The present author have no knowledge of such models in the literature. It seems therefore as a reasonable strategy to start modelling at a simple level and gradually increase the complexity.

\section{ONE-SUBGRAIN model: one axis of rotation}

A system consisting of one subgrain surrounded by immobile neighbouring subgrains can reduce its energy by allowing the mobile grain to rotate into the lowest possible energy state. The boundaries surrounding the subgrain can be a mixture of high- and low-angle boundaries, or sub-boundaries only. During the rotation, the orientation of the subgrain relative to a fixed co-ordinate system can be determined by introducing one or more vectors of the subgrain, e.g. along crystallographic axes. Crystallographic symmetries must be accounted for separately if only one position vector is employed. 
In a first attempt, the subgrain may only be allowed to rotate around one axis. The model can thereafter be generalised to any rotation.

\section{One-dimensional subgrain}

The simplest model is the semi one-dimensional case: a one dimensional, mobile subgrain of length $l_{1}$ has two dimension-less sub-boundaries with energies equal to the specific sub-boundary energy, cf. Figure 1 . The total sub-boundary energy of the system, $\mathrm{E}_{1 \mathrm{D}}$, can thus be written:

$$
E_{1 D}=\gamma_{0}^{(s b)}+\gamma_{2}^{(s b)}
$$

where $\gamma_{0}^{(s b)}$ and $\gamma_{2}^{(s b)}$ are the specific sub-boundary energies of the dimension-less boundaries of Figure 1. $\gamma_{0}^{(s b)}$ and $\gamma_{2}^{(s b)}$ are determined by the angles of misorientation over the boundaries, $\theta_{0}$ and $\theta_{2}$. The orientation of the subgrain can be determined by the angle, $\psi$, between a position vector and a fixed axis. The subgrain will experience a force at each boundary which will result in two torques, $\mathrm{T}_{\mathrm{G} 0}$ and $\mathrm{T}_{\mathrm{G} 2}$, about an axis of rotation $\mathrm{G}$ which is the mass centre with distance $l_{1} / 2$ from any sub-boundary. The sum of these torques will determine the rotation and can be written:

$$
\Sigma \mathrm{T}_{\mathrm{G}}=\frac{l_{1}}{2}\left(\frac{\partial \gamma_{0}^{(\mathrm{sb})}}{\partial \psi}+\frac{\partial \gamma_{2}^{(\mathrm{sb})}}{\partial \psi}\right)
$$

From classical mechanics (cf. e.g. Meriam and Kraige 1993):

$$
\Sigma \mathrm{T}_{\mathrm{G}}=\frac{\mathrm{dH}_{\mathrm{G}}}{\mathrm{dt}}
$$

where $\mathrm{H}_{\mathrm{G}}$ is the angular momentum of the mobile subgrain. The angular momentum of the mass system about the mass centre $G$ is the sum of the moments of the linear moments about $G$ of the total number of atoms, $N_{A}$, and is

$$
\mathrm{H}_{\mathrm{G}}=\sum_{\mathrm{j}=1}^{\mathrm{N}_{\mathrm{A}}}\left(\mathrm{r}_{\mathrm{j}} \cdot \mathrm{m}_{\mathrm{j}} \mathrm{v}_{\mathrm{j}}\right)
$$

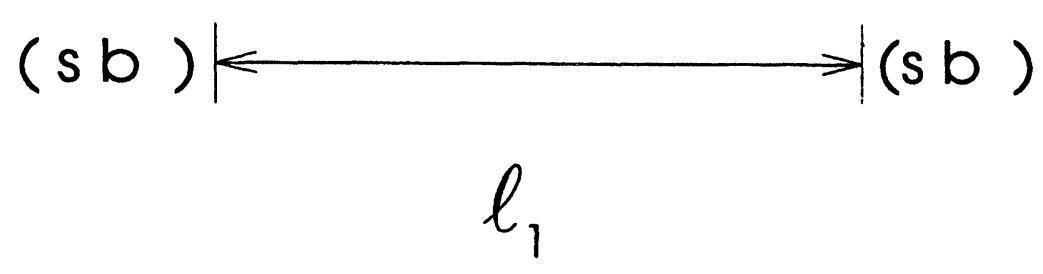

Figure 1 The principle of a semi one-dimensional subgrain. The subgrain of length $l_{1}$ has two subboundaries (sb) with energy equal the specific sub-boundary energy. The orientation of the subgrain, $\psi$, is the angle between a subgrain vector and a fixed axis. 
where $v_{j}$ is the atomic velocity, $m_{j}$ is the atomic mass and $r_{j}$ is the radial distance from the mass centre of the $j$ 'th atom. It is assumed that the subgrain consists of only one kind of atom with mass $\mathrm{m}_{\mathrm{A}}$. Introducing the angular velocity, $\omega=d \psi / d t$, Eq. (4) can be written:

$$
\mathrm{H}_{\mathrm{G}}=\mathrm{m}_{\mathrm{A}} \sum_{\mathrm{j}=1}^{\mathrm{N}_{\mathrm{A}}}\left(\mathrm{r}_{\mathrm{j}}^{2} \cdot \omega_{\mathrm{j}}\right)
$$

It is then assumed that all atoms have the same angular velocity, $\omega$, and that the atoms are closely packed on a line. In the case of an odd number of atoms and one atom at $G$, the sum of the squared distances from $G$ to each atom can, due to symmetry, be written (cf. Figure 2):

$$
\sum_{j=1}^{N_{A}} r_{i}^{2}=2 \frac{N_{A}-1}{2} \sum_{j=1}^{2}
$$

Hence,

$$
\sum_{j=1}^{\frac{N_{A}-1}{2}} r_{i}^{2}=r_{1}^{2}+r_{2}^{2}+r_{3}^{2}+\cdots=r_{1}^{2}+2^{2} r_{1}^{2}+3^{2} r_{1}^{2}+\cdots=r_{1}^{2} \sum_{j=1}^{\frac{N_{A}-1}{2}} j^{2}
$$

where $r_{1} / 2$ is the atomic radius $\left(r_{1} \cdot N_{A}=l_{1}\right)$. Introducing the notation $\sum_{j=1}^{\left(N_{A}-1\right) / 2} j^{2}=\Sigma$, Eq. (5) can now be written:

$$
\mathrm{H}_{\mathrm{G}}=2 \mathrm{~m}_{\mathrm{A}} \omega \frac{l_{1}^{2} \Sigma}{\mathrm{N}_{\mathrm{A}}^{2}}
$$

In a simple one-dimensional model, where atoms are not allowed to enter or exit the subgrain, the angular velocity, $\omega=d \psi / d t$, is the only variable. The rotation is then determined by combining Eqs. (2), (3) and (8):

$$
\frac{\mathrm{d} \omega}{\mathrm{dt}}=\frac{\mathrm{N}_{\mathrm{A}}^{2}}{4 l_{1} \mathrm{~m}_{\mathrm{A}} \Sigma}\left(\frac{\partial \gamma_{0}^{(\mathrm{sb})}}{\partial \psi}+\frac{\partial \gamma_{2}^{(\mathrm{sb})}}{\partial \psi}\right)
$$

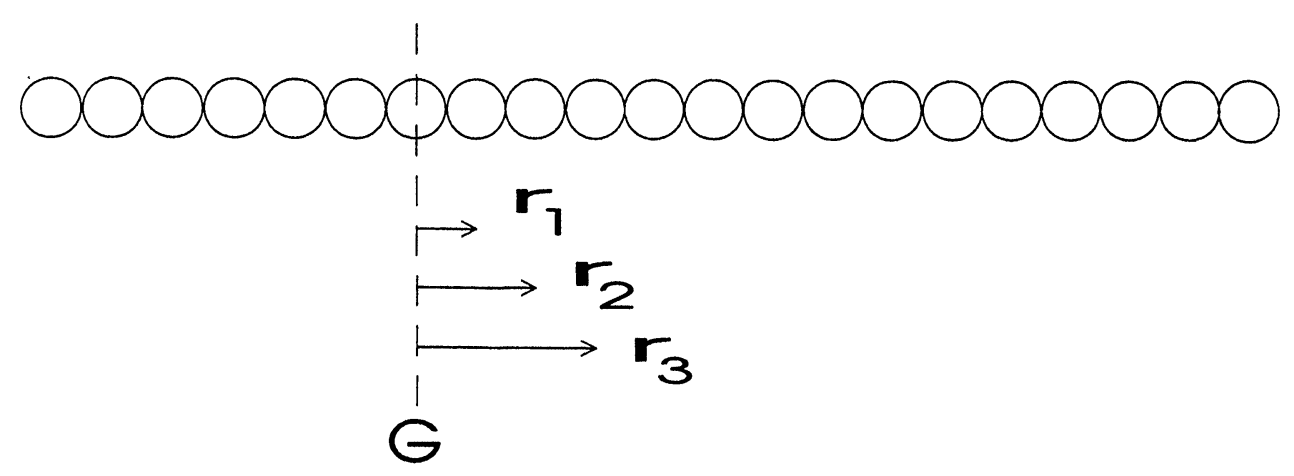

Figure 2 Part of the semi one-dimensional subgrain, showing atoms around the mass centre, G. One atom is positioned at $G$ and hence, $r_{0}=0$. The distance to the next atom is $r_{1}$, which is equal to one atomic diameter. 
Eq. (9) requires that the specific energies of the sub-boundaries can be found as a function of the angles of misorientation, $\theta_{0}$ and $\theta_{2}$. Generally, numerical solutions may be the only option. Read and Shockley (1950) derived the following well-known equation for simple tilt-boundaries:

$$
\gamma_{1}^{(\mathrm{sb})}=\frac{\gamma_{\max }}{\theta_{\max }} \theta_{i} \ln \frac{\mathrm{e} \theta_{\max }}{\theta_{i}}
$$

where $\theta_{\mathrm{i}}$ is the angle of misorientation of the i'th sub-boundary and $\theta_{\max }$ and $\gamma_{\max }$ are constants. Thus,

$$
\frac{\partial \gamma_{1}^{(\mathrm{sb})}}{\partial \theta_{1}}=\frac{\gamma_{\max }}{\theta_{\max }} \quad\left(\ln \frac{\mathrm{e} \theta_{\max }}{\theta_{1}}-1\right)
$$

which yields the wanted force term $\partial \gamma_{i}^{(\mathrm{sb})} / \partial \psi$ by converting from the angle of misorientation of a sub-boundary to the angle of orientation of the mobile subgrain. Numerically, it is possible to calculate an incremental change in orientation, $\Delta \psi$, due to a time increment, $\Delta t$. A discretised form of Eq. 9 yields the change in angular velocity:

$$
\Delta \omega=\frac{\mathrm{N}_{\mathrm{A}}^{2}}{4 l_{1} \mathrm{~m}_{\mathrm{A}} \Sigma}\left(\frac{\partial \gamma_{0}^{(\mathrm{sb})}}{\partial \psi}+\frac{\partial \gamma_{2}^{(\mathrm{sb})}}{\partial \psi}\right) \cdot \Delta \mathrm{t}
$$

and,

$$
\psi_{\mathrm{t}}=\psi_{\mathrm{t}-\Delta \mathrm{t}}+\left(\omega_{\mathrm{t}-\Delta \mathrm{t}}+\Delta \omega\right) \Delta \mathrm{t}
$$

\section{Two-dimensional subgrain}

A computer simulator for the one-dimensional model can yield interesting results, although the physical interpretation is restricted to the simplicity of such models. Several modifications can be introduced, e.g. stacking order of the atoms as a function of $\psi$, and the effect of replacing the Read-Shockley sub-boundary with other sub-boundaries. However, a two-dimensional model will represent the physics in a more realistic manner, without having the complexity of a three-dimensional model. The following model is restricted in that the total mass of the rotating subgrain is constant. Consequently, atoms are not allowed to enter or exit the mobile subgrain. Moreover, the sub-boundaries surrounding the subgrain is immobile, i.e. the only reduction possible of a subboundary's energy is by a reduction of the angle of misorientation due to rotation of the crystallographic lattice of the mobile subgrain.

Two-dimensional modelling introduces the requirement of mass transfer, cf. Figure 3. A non-circular subgrain cannot rotate unless a migration of atoms takes place. This is illustrated in Figure 3 by rotating a regular 6-sided subgrain about the centre of gravity an angle $\Delta \psi$. The position after rotation is indicated by broken lines. The triangles marked $v$ are voids and $e$ are excess material. The triangle areas are identical. Consequently, in order to facilitate the rotation, atoms must migrate from the $e$ areas to the $v$ areas during the rotation. Similarly to the one-dimensional subgrain, the subgrain will experience forces at the sub-boundaries. In addition, there will be forces which counters the rotation due to the misfit. These forces will be called geometrical friction forces, GFF, and are directed against the direction of rotation. The GFF will be zero for a circular subgrain rotating about the mass centre. Since mass transport is diffusion controlled, GFF will be strongly temperature dependent. GFF will also depend on the shapes of the subgrain. When comparing n-sided regular polygonal subgrains rotating 


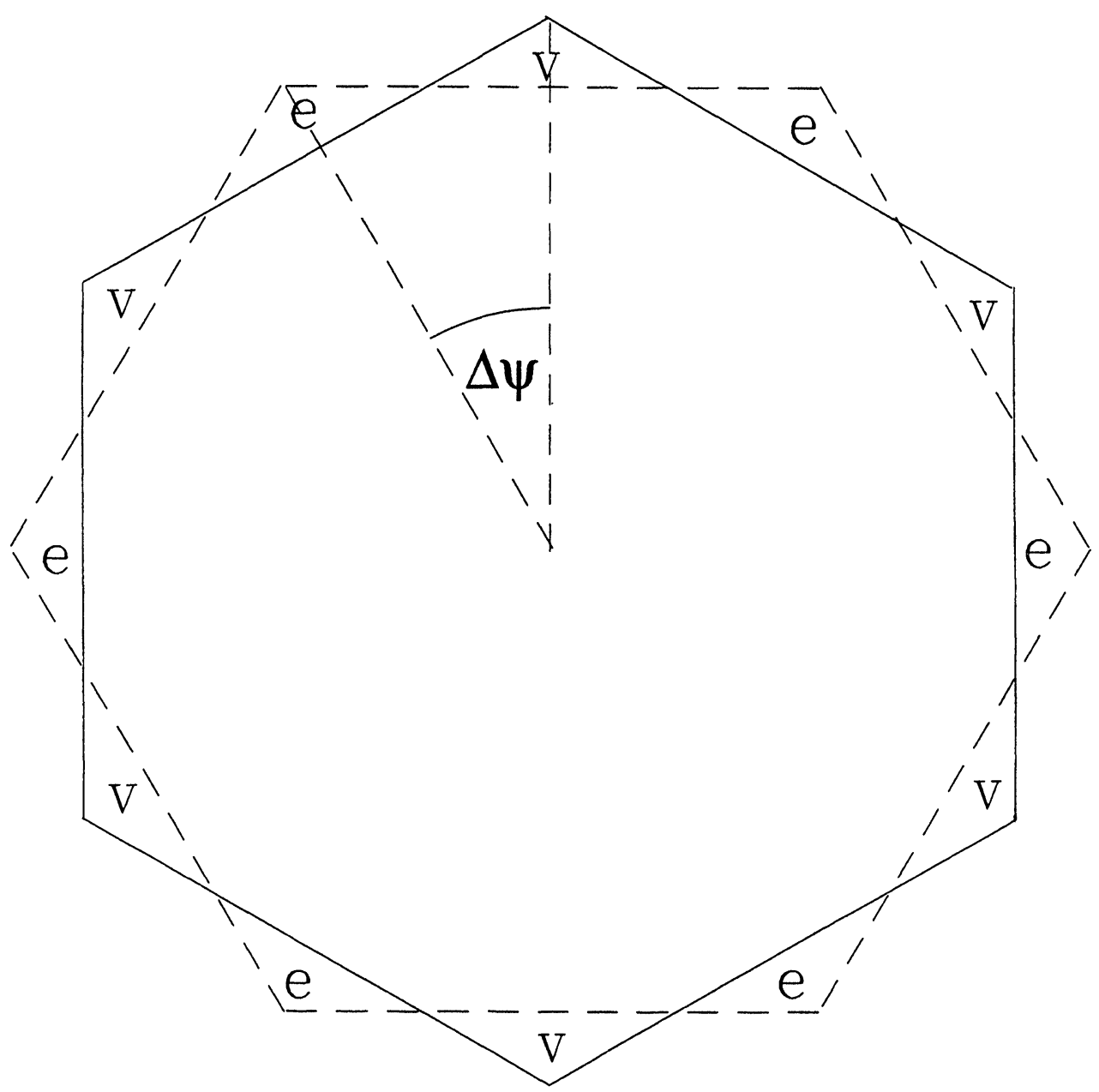

Figure $3 \mathrm{~A}$ six-sided regular polygonal-shaped subgrain is rotated an angle $\Delta \psi$. The sub-boundaries, solid lines, are immobile. The broken lines indicate the position of the subgrain after the rotation. Due to misfit, the triangles marked $e$ contain excess material whereas triangles marked $v$ are voids. Hence, in order to facilitate the rotations, mass transfer must take place. The diffusion of atoms into the voids will be highly temperature dependent and, consequently, subgrain coalescence will be highly temperature dependent.

about the centre of mass, GFF $\rightarrow 0$ as $n \rightarrow \infty$. GFF reaches the maximum value for small $n$-values. In addition to GFF, there may exist other forces directed against the direction of rotation. A change in the angle of misorientation will result in a change in the distance of separation between the dislocations in the sub-boundary. This can be achieved by dislocation climb. However, dislocation climb will require the diffusion of vacancies and, consequently, energy. It is therefore suggested to introduce boundary friction forces, BFF, to enable the modelling of these effects. The sum of the torques can thus be written: 


$$
\Sigma \vec{T}_{G}=\sum_{i=1}^{N_{s b}}\left(\vec{r}_{i}^{(s b)} \times \vec{F}_{i}^{(s b)}\right)+\sum_{i=1}^{N_{s b}}\left(\vec{r}_{i}^{(B F F)} \times \vec{F}_{i}^{(B F F)}\right)+\sum_{i=1}^{N_{G F F}}\left(\vec{r}_{i}^{(\text {GFF })} \times \vec{F}_{i}^{(G F F)}\right)
$$

where $N_{s b}$ is the number of sub-boundaries, $N_{G F F}$ is the number of GFF points. $\vec{r}_{i}^{(s b)}$ is the distance from the mass centre $G$ to $\vec{F}_{i}^{\left({ }^{\prime} b\right)}$ which is the force representing the $i$ 'th subgrain with scalar magnitude:

$$
\left|\vec{F}_{i}^{(s b)}\right|=L_{i} \frac{\partial \gamma_{i}^{(s b)}}{\partial \psi}
$$

where $L_{i}$ is the length of the sub-boundary. The direction of $\vec{F}_{i}^{(s b)}$ is determined by the sub-boundary energy as a function of the misorientation. $\overrightarrow{\mathrm{F}}_{i}^{(\mathrm{sb})}$ is thus directed towards the rotation that will reduce the sub-boundary energy of that particular boundary. $\vec{F}_{i}^{(\text {sb) }}$ may, in detailed models, have to be replaced by a series of forces representing segments of the sub-boundary. $\vec{r}_{i}^{(B F)}$ is the distance from $G$ to $\vec{F}_{i}^{(B F F)}$ which is the boundary friction force of the $i$ 'th sub-boundary; directed against the direction of rotation. $\vec{r}_{i}^{\text {(GF) }}$ is the distance from the mass centre $G$ to $\vec{F}_{i}^{(G F F)}$ which is the force representing the $i$ 'th GFF point.

Eg. (14) should represent the physics, and the present form of the equation is only valid if the sub-boundaries can be represented by one vector of force due to the subboundary energy and one force due to the boundary friction, and if the GFF can be modelled as a series of discrete forces, preferably linked to the flux of vacancies, acting along the sub-boundaries. The angular momentum of the mobile subgrain is given by Eq. (4) in vector form:

$$
\overrightarrow{\mathrm{H}}_{\mathrm{G}}=\sum_{\mathrm{j}=1}^{N_{\mathrm{A}}}\left(\overrightarrow{\mathrm{r}}_{\mathrm{j}} \times \mathrm{m}_{\mathrm{j}} \overrightarrow{\mathrm{v}}_{\mathrm{j}}\right)
$$

Employing similar arguments as for the one-dimensional case yields:

$$
\overrightarrow{\mathrm{H}}_{\mathrm{G}}=\mathrm{m}_{\mathrm{A}} \sum_{\mathrm{j}=1}^{\mathrm{N}_{\mathrm{A}}}\left[\overrightarrow{\mathrm{r}}_{\mathrm{j}} \times\left(\vec{\omega} \times \overrightarrow{\mathrm{r}}_{\mathrm{j}}\right)\right]
$$

which can be written:

$$
H_{G}=m_{A} \sum_{j=1}^{N_{A}}\left[\left|\vec{r}_{j}\right|^{2} \vec{\omega}-\vec{r}_{j} \cdot \vec{\omega} \vec{r}_{j}\right)
$$

The second term is zero if the angle between $\vec{r}$ and $\vec{\omega}$ is 90 degrees. Differentiation yields:

$$
\frac{d \vec{H}_{G}}{d t}=m_{A}\left(\vec{\omega} \sum_{j=1}^{N_{A}} \frac{d\left|\vec{r}_{j}\right|^{2}}{d t}+\frac{d \vec{\omega}}{d t} \sum_{j=1}^{N_{A}}\left|\vec{r}_{j}\right|^{2}\right)
$$

If the radial distances to the atoms are constant, the first term is zero. Hence,

$$
\frac{\mathrm{d} \vec{\omega}}{\mathrm{dt}}=\frac{1}{\mathrm{~m}_{\mathrm{A}} \sum_{j=1}^{N_{\mathrm{A}}}\left|\overrightarrow{\mathrm{r}}_{j}\right|^{2}} \frac{\mathrm{d} \overrightarrow{\mathrm{H}}_{\mathrm{G}}}{\mathrm{dt}}
$$

By combination with Eqs. (3) and (14):

$$
\frac{\mathrm{d} \vec{\omega}}{\mathrm{dt}}=\frac{1}{\mathrm{~m}_{\mathrm{A}} \sum_{j=1}^{N_{A}}\left|\overrightarrow{\mathrm{r}}_{j}\right|^{2}}\left[\sum_{i=1}^{N_{s b}}\left(\overrightarrow{\mathrm{r}}_{i}^{(\mathrm{sb})} \times \overrightarrow{\mathrm{F}}_{i}^{(\mathrm{sb})}\right)+\sum_{\mathrm{i}=1}^{N_{\mathrm{sp}}}\left(\overrightarrow{\mathrm{r}}_{i}^{(\mathrm{BFF})} \times \overrightarrow{\mathrm{F}}_{\mathrm{i}}^{(\mathrm{BFF})}\right)+\sum_{i=1}^{N_{\mathrm{GFF}}}\left(\overrightarrow{\mathrm{r}}_{i}^{(\mathrm{GFF})} \times \overrightarrow{\mathrm{F}}_{i}^{(\mathrm{GFF})}\right)\right]
$$


Eq. (21) can be discretised and the incremental change in orientation, $\Delta \vec{\psi}$, due to a time increment, can be determined in the same manner as shown for the one-dimensional subgrain.

A different modelling approach was taken in Saetre and Ryum (1995). By assuming that the migration of vacancies was rate controlling, an equation for $\mathrm{d} \psi / \mathrm{dt}$ was derived, discretised and employed in a computer model for two-dimensional subgrain coalescence.

\section{DISCUSSION}

Eq. (21) was derived by employing formal vector operations. Hence, the equation can be similarly valid for a three-dimensional subgrain with a rotation in space and governed by the laws of physics. However, many details in the two-dimensional case has not been worked out, e.g. the derivation of the $\vec{F}_{i}^{(\text {GFF }}$ forces. It is thus argued that several workable two-dimensional models should be demonstrated before three-dimensional modelling is undertaken. It is seen that $\mathrm{d} \vec{\omega} / \mathrm{dt}$ is inversely proportional to the sum of the radial distances from the centre of mass and to the atoms. Hence, the smaller subgrains are the most likely candidates for rotation. The GFF term, which is directed against the direction of rotations, indicates that the shape of the subgrain is a determining factor for rotation. A highly regular subgrain is more likely to rotate than an irregular one. It should be pointed out that the two last $\Sigma$-terms are forces working against a rotation. Hence, the first $\Sigma$-term in the brackets has to overcome those forces in order for a rotation to take place. Even this $\Sigma$-term contains forces directed against the rotation. Normally, during a rotation, the angle of misorientation of some boundaries will increase while other boundaries obtain a reduction in the angle of misorientation. Since the two last $\Sigma$-terms are dependent on the diffusion of vacancies, they will be reduced at higher temperatures, thus increasing the possibility for subgrain coalescence.

The generalisation from one mobile subgrain to an ensemble of subgrains is quite straightforward as demonstrated previously (Saetre, Ryum and Evangelista, 1991; Saetre and Ryum, 1995). However, models which include the migration of the sub-boundaries will substantially increase the level of details and complexity of the model.

In conclusion, $\mathrm{Hu}$ (1981) reviewed the state of knowledge of subgrain coalescence and found that both experimentally and theoretically, more research was needed. The present author have suggested a new modelling approach based on classical dynamics. It has been shown that 3-dimensional modelling of subgrain coalescence is possible. However, more research should be carried out with two-dimensional simulators before a detailed modelling in three-dimensions is undertaken. The present model indicates that small and regular subgrains are the most likely candidates for rotation.

\section{References}

Fujita, H. (1961). Direct observation of Subgrain-Growth of Cold-Worked Aluminium by means of Electron Microscopy. Journal of the Physical Society of Japan, 16, 397-406.

$\mathrm{Hu}$, Hsun. (1962). Direct observations on the annealing of a Si-Fe crystal in the electron microscope. Transactions of the Metallurgical Society of AIME, 224, 75-84.

$\mathrm{Hu}$, Hsun. (1963). Annealing of Silicon-Iron Single Crystals. In Recovery and Recrystallization of Metals, edited by L. Himmel, pp. 311-378. New York: Interscience.

Hu, Hsun. (1981). Recovery, Recrystallisation and Grain Growth. In Metallurgical Treatises, edited by J. Tien and J. F. Elliott, pp. 385-407. Metallurgical Society of AIME.

$\mathrm{Hu}$, Hsun. (1993). Private communication to the present author. 
Li, J. C. M. (1962). Possibility of Subgrain Rotation during Recrystallization. Journal of Applied Physics, 33, 2958-2965.

Meriam, J. L. and Kraige, L. G. (1963). Engineering Mechanics, 3rd edn., New York: John Wiley $\&$ Sons.

Read, W. T. and Shockley, W. (1950). Dislocation Models of Crystal Grain Boundaries. Physical Review, 78, 275-289.

Saetre, T. O., Ryum, N. and Evangelista, E. (1991). Simulation of Subgrain Growth by Subgrain Rotation: A One-Dimensional Model. Metallurgical Transactions A, 22A, 2257-2263.

Saetre, T. O. and Ryum, N. (1995). On Grain and Subgrain Rotations in Two Dimensions. Metallurgical and Materials Transactions A, 26A, 1687-1697. 